\title{
LOS BIOCOMBUSTIBLES DESDE LA PERSPECTIVA DEL COMERCIO INTERNACIONAL Y DEL DERECHO DE LA ORGANIZACIÓN MUNDIAL DEL COMERCIO
}

\author{
Juan Pablo Arístegui Sierra*
}

\begin{abstract}
RESUMEN
El surgimiento de un mercado internacional de biocombustible es el resultado de determinadas politicas de los países que buscan grados relativos de independencia energética así como el cumplimiento de sus compromisos ambientales. Sin embargo, los riesgos para la seguridad alimentaria mundial y las amenazas sobre el medio ambiente han llamado la atención de la comunidad internacional que reclama una producción y comercio sustentable. El comercio internacional de este producto y el derecho de la Organización Mundial del Comercio cumplen un rol en la disciplina de los biocombustibles que merecen ser destacados. Los problemas fundamentales dicen relación, inter alia, con la "clasificación" de este producto, los subsidios y los estándares domésticos.
\end{abstract}

\section{BIOCOMBUSTIBLES - COMERCIO INTERNACIONAL - DESARROLLO SOSTENIBLE}

\section{Biofuels from the international trade perspective and the law of the WTO}

\begin{abstract}
The rise of the international market of biofuels is the result of specific politics of countries that are searching relative degrees of energy independence as well as fulfillment of their environmental commitments. However, the new risk for global food security and the threats over the environment has called the attention of the international community that claim for a sustainable production and trade. The international trade of this product and the law of the WTO play a role in the discipline of biofuels that is worth to be highlighted. The fundamental issues, inter alia, are related to "classification" of this product, subsidies and domestic standards.
\end{abstract}

\section{BIOFUELS - INTERNATIONAL TRADE - SUSTAINABLE DEVELOPMENT}

* Licenciado en derecho, profesor de Derecho Internacional Público y Relaciones Internacionales, Universidad Pompeu Fabra, Barcelona, España, y en la Universidad Diego Portales, Santiago de Chile. juanpablo.aristegui@upf.edu Artículo recibido el 2 de diciembre de 2008 y aceptado para su publicación por el Comité Editorial el 18 de mayo de 2009. 


\section{INTRODUCCIÓN}

$\mathrm{L}$ as políticas de los países dirigidas a alcanzar grados relativos de independencia energética así como de lograr los compromisos internacionales en materia ambiental, en particular del cambio climático, han sido fundamentales para el surgimiento de un mercado de los biocombustibles; mercado que se encuentra en sus primeros estadios y se localiza principalmente a nivel nacional o regional.

Cuatro áreas políticas interrelacionadas deben ser tenidas en cuenta para analizar el problema de los biocombustibles: la energía, la alimentación y la agricultura, el medio ambiente y el comercio.

El comercio internacional de los bicombustibles ha favorecido hasta cierto punto que dichas políticas sobrepasen las fronteras nacionales para ubicar el problema dentro del plano internacional. Los aspectos más problemáticos de la producción y del comercio internacional de esta nueva fuente de energía dicen relación principalmente con los riesgos para la seguridad alimentaria y con el impacto ambiental.

La Organización de las Naciones Unidas para la Agricultura y la Alimentación -FAO- ha descrito tres escenarios posibles frente a lo anterior: seguir como hasta ahora, lo que implica proseguir el camino iniciado; establecer una moratoria que prohíba temporalmente la producción; ${ }^{1}$ y el desarrollo de un consenso intergubernamental sobre los biocombustibles sostenible. $^{2}$

Esto último, es un llamado al Derecho internacional general y sectorial para poner orden en la interrelación de las cuatro políticas antes mencionadas. Sin embargo, no hay claridad acerca de cuál sea el foro más indicado ni tampoco de si existe la voluntad política suficiente en demanda de un nuevo régimen internacional, dadas las ventajas relativas y absolutas que se encuentran en juego. ${ }^{3}$

El Sistema Multilateral del Comercio y el derecho de la Organización Mundial del Comercio -OMC- tienen un rol evidente en la disciplina de este nuevo mercado. Sin embargo, el sector de los biocombustibles no ha sido tratado de manera directa en el seno de la organización.

La Conferencia Ministerial de Doha de 2001, que ha dado paso a la denominada Ronda del Desarrollo, ha puesto en la agenda de la OMC el compromiso del comercio

${ }^{1}$ La demanda de una moratoria no sólo tiene origen en los países más afectados en el último tiempo por la subida en los precios internacionales de los alimentos -como es el caso del continente africano-, sino que ha sido solicitada también por el ex Relator Especial sobre el Derecho a la Alimentación de las Naciones Unidas -Jean Ziegler-, quien ha llamado a una prohibición de al menos cinco años para los biocombustibles de primera generación.

${ }^{2}$ FAO, Bioenergía, Seguridad y Sostenibilidad Alimentarias: Hacia el Establecimiento de un Marco Internacional (Roma: FAO, 2008. Doc. HLC/08/INF/3).

3 Junto a la FAO, existen hoy diversas iniciativas internacionales ocupadas en el tema, algunas de ellas bajo el alero de las Naciones Unidas: La Asociación mundial de la bioenergía (GBEP); la Mesa redonda sobre los biocombustibles; el Foro Internacional de Biocombustibles; y el mecanismo interinstitucional de las Naciones Unidas, ONU-Energía. 
internacional con el desarrollo y actualizó y llenó de contenido el objetivo de la organización en cuanto a incorporar dentro de su sistema el concepto de desarrollo sostenible. ${ }^{4}$

En consecuencia, el concepto de desarrollo sostenible, acuñado en la Conferencia de Naciones Unidas sobre el Medio Ambiente y el Desarrollo de 1992, ${ }^{5}$ debería ser tomado en cuenta al momento de examinar las reglas del sistema OMC allí donde sea pertinente. En particular, cabe adelantar que el desarrollo sostenible en el ámbito comercial está íntimamente relacionado con los métodos de proceso y producción (PPMs por sus siglas en inglés), así como con el enfoque analítico del ciclo de vida de los productos. ${ }^{6}$ En ambos casos el derecho de la OMC tendrá que acomodarse para dar cabida a dichos enfoques, porque hasta el momento el principio de no discriminación ha impedido un reconocimiento abierto sobre este tipo de cuestiones en la jurisprudencia de la organización, toda vez que pueden ser utilizadas como una restricción encubierta al comercio internacional. ${ }^{7}$ Por otro lado, ambos enfoques resultan oportunos para enfrentar los impactos ambientales así como los riesgos en la disponibilidad y acceso a los alimentos derivados de unos patrones de producción determinados en el sector de los biocombustibles.

El objetivo de este artículo es aproximarse a la cuestión de los biocombustibles desde la perspectiva del comercio internacional y del derecho de la OMC con el objeto de examinar el estado actual del problema y de introducir, ahí donde sea pertinente, la noción de desarrollo sostenible.

\section{Políticas detrás de los biocombustibles. Su producción Y COMERCIO. SEgURIDAD ALIMENTARIA E IMPACTO AMBIENTAL}

\section{A. Los objetivos políticos detrás del surgimiento de los biocombustibles}

Se ha señalado que mientras el actual interés de los Estados Unidos en los biocombustibles responde a su deseo de alcanzar mayores grados de seguridad energética y

${ }^{4}$ A diferencia del GATT de 1947, el Acuerdo de Marrakech en virtud del cual se crea la OMC contempla en su preámbulo el objetivo de un desarrollo sostenible en relación con la utilización (óptima, señala el acuerdo) de los recursos mundiales. Al respecto, véase: Declaración Ministerial de 14 de noviembre de 2001. OMC Doc. WT/MIN(01)/DEC/1 de 20 de noviembre de 2001.

${ }^{5}$ El concepto en sí ya había sido definido por la Comisión Brundtland en el informe Nuestro Futuro Común de 1987; sin embargo, fue durante la Cumbre de la Tierra (nombre con que se conoce la CNUMAD) donde el concepto alcanzó plena difusión y operatividad.

${ }^{6}$ Al efecto, el Principio 8 de la Declaración de Río señala: "Para alcanzar el desarrollo sostenible y una mejor calidad de vida para todas las personas, los Estados deberían reducir y eliminar las modalidades de producción y consumo insostenibles y fomentar políticas demográficas apropiadas”.

${ }^{7} \mathrm{Al}$ respecto, cabe recordar la decisión del Grupo Especial del GATT en el caso "Atún-Delfines" entre los Estados Unidos y México, en donde la legislación de aquel imponía restricciones a la importación de atún cuando las técnicas de pesca utilizadas no pusieran a salvo a los delfines. Al respecto, el Grupo Especial concluyó que “...un país no puede restringir sus importaciones referidas a un producto únicamente porque procede de un país que aplica una política ambiental diferente”, de lo contrario, se argumentó, se estaría dando una aplicación extraterritorial de la legislación norteamericana. GATT BISD DS29/R de 16 de junio de 1994. 
reducir su dependencia respecto del petróleo proveniente de Medio Oriente, el interés de Europa radica principalmente en consideraciones ambientales y su intento por cumplir con el Protocolo de Kyoto. ${ }^{8}$

En los Estados Unidos la Energy Policy Act de $2005^{9}$ ha impuesto como meta alcanzar 7,5 billones de galones de biocombustible para el año 2012 (lo que se conoce como Renewable Fuel Standard), horizonte que será superado dentro de poco y que según predicciones del Departamento de Agricultura de los Estados Unidos alcanzará los 12 billones de galones para el año 2015. ${ }^{10}$

El Presidente Bush, por su parte, en el State-of-the Union de $2007^{11}$ hizo un llamado a aumentar exponencialmente la producción de etanol de modo de alcanzar una meta de 35 billones de galones para el año 2017, es decir, más del doble que las predicciones del propio Departamento de Agricultura, lo que ha sido fuertemente cuestionado desde diversos sectores norteamericanos que consideran que aún no existe un mercado capaz de absorber tal cantidad de etanol. ${ }^{12}$

En cambio, en Europa han sido principalmente las consideraciones ambientales las que inspiran la política en materia de energía. En la reunión de marzo de 2007 del Consejo Europeo, los biocombustibles fueron tratados bajo la rúbrica de la integrated climate and energy policy; ${ }^{13}$ es decir, para Europa, la política energética está contrapunteada por los desafíos del cambio climático y por lo tanto vinculada con los compromisos adoptados en el Protocolo de Kyoto.

Las Comunidades Europeas cuentan desde 2003 con una directiva ${ }^{14}$ relativa al fomento del uso de biocarburantes ${ }^{15} \mathrm{u}$ otros combustibles renovables en el transporte, que reitera, en su considerando 17, el objetivo apuntado en el Libro Verde relativo a la sustitución del 20 por ciento de los carburantes convencionales por carburantes alternativos en el sector del transporte por carretera desde la fecha de la directiva hasta el $2020 .{ }^{16}$

${ }^{8}$ International Energy Agency, World Energy Outlook 2006 (OECD/AIE 2006), pp. 385-416.

9 Texto completo de la ley en formato pdf en http://www.doi.gov/iepa/EnergyPolicyActof2005.pdf

${ }^{10}$ Paul C. Westcott, "U.S. Ethanol Expansion Driving Changes Throughout the Agricultural Sector", Amber Waves, U.S. Department of Agriculture, septiembre de 2007, p. 3. Disponible en: http://www.ers.usda. gov/AmberWaves/September07/Features/Ethanol.htm

112007 State-of-the-Union Policy Initiatives, en: www.whitehouse.gov/stateoftheunion/2007/ initiatives/ index.html.

${ }^{12}$ En esta dirección, la Energy Policy Research Foundation ha señalado que el mercado norteamericano no es capaz de absorber más de 15 billones de galones en una proyección a futuro de varios años, en particular, por las dificultades relativas a la flexibilidad del parque automotriz para funcionar con etanol así como a los problemas de transmisión que exige una nueva infraestructura que el país aún no cuenta. Véase: Energy Policy Research Foundation, Ethanol Part II: Is a Home-Grown Fuel Policy Undermining U.S. Energy Security?, 2007. Disponible en internet: http://www.eprinc.org/pdf/HomeGrownFuelUSEnergySecurity.pdf

${ }^{13}$ European Council, Presidency Conclusions (Brussels: European Council, $8 \pm 9$ March 2007), available at: <www.europa-eu-un.org/articles/it/article_6836_it.htm >.

${ }^{14}$ Directiva 2003/30/CE del Parlamento Europeo y del Consejo, de 8 de mayo de 2003.

${ }^{15} \mathrm{Si}$ bien existen diferencias técnicas entre los biocarburantes y los biocombustibles, para los efectos del presente artículo se utilizan ambas expresiones como sinónimos.

${ }^{16}$ Sobre el marco legal europeo en relación a los biocombustibles, véase: Delvaux, B., "Prompting Biofuels in Energy Supply the European Legal Framework”, en European Environmental Law Review, 2004, pp. 66-70. 
El caso de Brasil es diferente. Su interés en el etanol se inicia en los años 70 en respuesta a la primera crisis del petróleo y por lo tanto el desarrollo de la industria del etanol fue impulsado principalmente por motivos económicos. Las condiciones de la producción de etanol en Brasil hicieron posible competir con los altos precios del crudo. En la actualidad, Brasil se perfila como modelo no sólo por el éxito en la producción sino que también por la sostenibilidad de la misma en términos ambientales y alimentarios.

El resultado de las políticas mencionadas, más allá del origen o los motivos que las inspiran, ha hecho posible la creación de un mercado que probablemente no hubiera surgido de modo espontáneo. Las consecuencias en el comercio internacional serán evidentes dado que los Estados Unidos y la UE requerirán importar todo aquello que no sean capaces de producir. Por esta razón Brasil es actualmente el mayor promotor de un comercio internacional libre de barreras para los biocombustibles, con ventajas comparativas indiscutidas. ${ }^{17}$

\section{B. Panorama de la producción y del comercio de los biocombustibles}

\section{Producción de biocombustibles}

Los biocombustibles líquidos son el biodiésel y el bioetanol o etanol. ${ }^{18}$ El primero de ellos remplaza al diésel fósil, en tanto el etanol es un sustituto de la gasolina. Aquel es una especie de aceite, mientras que éste, una especie de alcohol. Las materias primas utilizadas en cada caso son, en consecuencia, diferentes además de variadas. ${ }^{19}$

Los costos de producción de los biocombustibles varían ampliamente de un proceso a otro y de región a región. Aunque está bien desarrollada la tecnología para producir etanol a partir de granos y cultivos azucareros, o biodiésel a partir de aceites vegetales, estas diferencias en los costos de producción se deben fundamentalmente a los costos de la materia prima, el tipo de energía usado (tanto calor como electricidad) y los precios obtenidos por los subproductos derivados durante el proceso de producción. ${ }^{20}$ En general, el etanol es más competitivo y se espera que siga siéndolo debido a que los costos de producción han caído mucho más rápido que los del biodiésel.

${ }^{17}$ United Nations Conference on Trade and Development, The Emerging Biofuels Market: Regulatory, Trade and Development Implications (Geneva: UNCTAD, 2006), p. 11.

18 También existen los biocombustibles en forma de gas, como el biogás. Sin embargo, el presente artículo se refiere a los biocombustibles líquidos dada su importancia frente a los gaseosos en el sector del transporte.

${ }^{19}$ Sobre la producción y exportación mundial de las materias primas de uno y otro biocombustible, ver: Almada, M., "Análisis de la producción de materias primas para la elaboración de bioetanol y biodiésel, y de estos combustibles, presente y esperada hasta 2020, en países potencialmente proveedores de Chile”, Estudio realizado en estadía profesional en la FAO, 2006. Disponible en: http://www.minagri.gob.cl/biocombustibles/documentos/analisis_produccion_etanol_biodiésel_paises_proveedores_chile.pdf

${ }^{20}$ Existe un mercado de los subproductos de la industria. Algunos animales, como los rumiantes, pueden alimentarse con granos destilados que es un subproducto de la producción de etanol, mientras que los cerdos y las aves no. 
Un factor importante para tomar en cuenta son los "precios umbral" del petróleo crudo a partir de los cuales la producción de biocombustibles se vuelve sostenible y competitiva. Los precios umbral, o precios mínimos, representan una estimación de aquellos precios del crudo con respecto a los cuales los precios nacionales libres de impuestos de la gasolina y del diésel son iguales a los costos de producción del etanol y biodiésel, respectivamente, tomando en consideración las diferencias en su contenido energético. $^{21}$

De acuerdo con la Agencia Internacional de Energía (AEI), los mayores productores de biocombustibles son Brasil, Estados Unidos, la Unión Europea, China, India y Canadá.

Mientras Brasil produce etanol principalmente a base de caña de azúcar, los Estados Unidos producen etanol principalmente a base de maíz amarillo y Europa a base de cereales y remolacha. Sin embargo, los países europeos son productores fundamentalmente de biodiésel, no de etanol, del cual el 90 por ciento del total se comercializa internamente y su participación en la producción mundial de biodiésel alcanza al 87 por ciento. Alemania y Francia son los mayores productores, y Alemania representa por sí sola el 62 por ciento del total de producción de biodiésel en Europa. La producción de etanol está igualmente concentrada entre los Estados Unidos y Brasil con el 80 por ciento del total de la producción mundial, del cual más del 50 por ciento corresponde a este último. Tras estos países, China e India son los mayores productores de biocombustible, en particular de etanol. Canadá, por su parte, con una participación menor, es productor esencialmente de biodiésel.

\section{Comercio de biocombustibles}

No obstante que el comercio de biocombustibles es predominantemente nacional o regional, el comercio internacional comienza a ganar terreno dados el incremento del transporte terrestre, las reducidas alternativas al uso de petróleo y gas en este sector y, muy fuertemente, debido a las metas de política energética impuestas por los países. Por lo tanto, a pesar de que en la actualidad los biocombustibles representan poco más del 1 por ciento de la demanda total de combustible para transporte (y entre el 4 y el 7 por ciento según las proyecciones de la AIE para el 2030 dependiendo del escenario), ${ }^{22}$ los biocombustibles parecen una buena oportunidad frente a los elevados precios del crudo internacional.

Comparando entre los tipos de biocombustible, el rasgo más sobresaliente es que mientras el etanol ha experimentado una fuerte expansión desde el 2000 a la fecha, ha habido muy poco comercio internacional de biodiésel en el mismo período. En efecto, del

\footnotetext{
${ }^{21}$ Instituto Interamericano de Cooperación para la Agricultura, Estrategias para construir una plataforma de cooperación horizontal sobre agroenergía y biocombustibles (IICA, 2006), pp. 30 y ss.

22 International Energy Agency, World Energy Outlook 2006 (en adelante WEO 2006),... op. cit. Los escenarios se refieren a la nomenclatura utilizada por la Agencia Internacional de Energía que distingue entre escenario de referencia y escenario político alternativo.
} 
total de biocombustible comercializado internacionalmente el etanol alcanza el 93 por ciento y el biodiésel lo que resta. Sin embargo, en cuanto a las materias primas o inputs de uno y otro, la situación es la inversa: mientras existe bastante comercio internacional de las materias primas del biodiésel, hay poco comercio internacional de las de etanol. ${ }^{23}$ Esto se explica fundamentalmente porque los países productores de etanol son a la vez grandes productores de las materias primas utilizadas para su elaboración.

Por su parte, los instrumentos de política comercial o de medidas que repercuten en el comercio de biocombustibles exhiben agudas diferencias dependiendo del país, del tipo de biocombustible y de las materias primas con que está hecho. ${ }^{24}$

En conclusión, parece claro que la demanda internacional continuará aumentando de forma sostenida y, por consiguiente, tanto Estados Unidos como la UE requerirán importar todo aquello que no sean capaces de producir localmente, lo que representa una oportunidad para los países en desarrollo productores ya sea de biocombustibles como de las materias primas con las que se producen. Sin embargo, los elevados aranceles, los fuertes subsidios así como las medidas técnicas y las eventuales exigencias de certificación desafían el sistema multilateral de comercio en cuanto a que pueden entenderse como barreras comerciales que impiden o retrasan la creación del sector en los países que cuentan con ventajas comparativas.

\section{Polémicas que rodean a los biocombustibles: la seguridad alimentaria y el impacto ambiental}

\section{La seguridad alimentaria y los biocombustibles}

El impacto de los biocombustibles en la seguridad alimentaria se ha limitado al debate rotulado "alimentos, piensos o combustibles" (food, feed or fuel); debate que representa la cara más mediática del problema. ${ }^{25}$ Sin embargo, el problema trasciende a cuestiones más complejas. De acuerdo con la Comisión sobre Desarrollo Sostenible de las Naciones Unidas, dicha discusión "llega a ser demasiado simplista y no logra

${ }^{23}$ Cabe advertir la dificultad en la recolección de estadísticas pertinentes, entre otras razones, porque uno y otro biocombustible tiene una clasificación aduanera diferente dentro del Sistema Armonizado de la Organización Mundial de Aduanas según veremos infra, que hace complejo averiguar acerca de si las materias primas comercializadas son utilizadas para la producción de biocombustible o para alimento humano o animal. Al respecto, véase: United Nations Conference on Trade and Development, The Emerging Biofuels Market... op. cit.

${ }^{24}$ Sobre las medidas que repercuten en el comercio de biocombustibles tanto en los Estados Unidos como en la UE así como otros países productores y el impacto en los países en desarrollo, véase: Jank Marcos, et al., EU and US Policies on Biofuels: Potential Impacts on Developing Countries (Washington, D.C.: German Marshall Fund of the United States, 2007).

${ }^{25}$ La consigna más escuchada en la región respecto al problema alimentario ha sido el grito de lucha mexicano "sin maíz, no hay país"; producto con el cual se hace la tortilla y que representa una de las fuentes de alimentos básicos de la cultura azteca. Como ésta, se han repetido decenas de manifestaciones y revueltas callejeras por diversos países africanos y de Asia en protesta por el hambre y la falta de acceso a los alimentos. 
reflejar la total complejidad de los factores que determinan la seguridad alimentaria en un lugar o momento dados". ${ }^{26}$

Para un auténtico análisis sobre las posibles consecuencias de la expansión de la producción de biocombustibles líquidos -lo cual excede los objetivos de este artículo-, habría que examinar cómo se ven afectadas las cuatro dimensiones de la seguridad alimentaria: disponibilidad, acceso, estabilidad y utilización. No obstante, veremos brevemente algunos aspectos que dicen relación con la disponibilidad y el acceso.

La disponibilidad consiste en el suministro adecuado de los alimentos, el cual se verá más o menos afectado en función del modo en que se utilicen y destinen la tierra, el agua y otros recursos. Si la utilización de dichos recursos es "desviada" o atraída por el sector de los biocombustibles, no se encontrarán disponibles para la producción de alimentos y comienza así la competencia entre los cultivos energéticos y los cultivos alimentarios. De acuerdo con la AIE, 14 millones de hectáreas en el mundo -correspondientes al 1 por ciento de la tierra cultivable- están actualmente destinadas a la producción de biocombustibles y se espera que para el año 2030 alcance el 2,5 o 3,8 por ciento del total de tierras cultivables según el escenario que se utilice como referencia. En cualquier caso, dichos porcentajes, aunque aparentemente bajos, tienen efectos prácticos de consideración.

El acceso, por su parte, se refiere a la posibilidad de las familias u hogares de producir o comprar suficiente comida para sus necesidades. El acceso está influenciado directamente por los ingresos de las familias y los precios de los alimentos. Si la producción de biocombustibles conduce a subir los precios de los commodities el acceso podría verse comprometido para aquellas familias de bajos ingresos, al menos en el corto plazo.

Los precios internacionales de los alimentos han experimentado fuertes subidas en los últimos años. Los biocombustibles, la mayor demanda de países en desarrollo como China e India (cambios en la dieta) y las diversas sequías que han asolado distintos puntos del planeta (como el caso de Australia en 2007) explican este aumento que deja a su paso serios problemas de hambruna y revueltas en la población. Resulta difícil averiguar la incidencia de cada una de estas causas, pero lo que está fuera de duda es que mientras las sequías resultan poco controlables y casi son impredecibles y la mayor demanda de alimentos en países en desarrollo es un signo positivo y no negativo, lo único corregible son las políticas que inspiran la producción de biocombustibles que han develado la creación de riesgos no previstos como los derivados de la competencia con los cultivos agrícolas.

Los Estados Unidos han sido tradicionalmente el mayor exportador de maíz con una participación de entre el 60-70 por ciento del total de las exportaciones mundiales. Tras el surgimiento de la industria del etanol a base de maíz, y de acuerdo con predicciones del propio Departamento de Agricultura, la cuota de los Estados Unidos en las

${ }^{26}$ United Nations Commission on Sustainable Development, Sustainable Energy: A Framework for DecisionMakers (Mayo 2007). Disponible en internet: http://esa.un.org/un-energy/pdf/susdev.Biofuels.FAO.pdf 
exportaciones de maíz puede caer hasta el 55 por ciento. ${ }^{27}$ Esto es consecuencia, en gran medida, de la desviación desde el sector exportador hacia el sector del etanol, ${ }^{28}$ lo que no solo repercute en el mercado internacional sino que también en el interno, aumentando los precios del maíz y de los productos derivados, los precios de otros granos como la soja que han cedido paso frente a los cultivos de maíz, así como de los productos ganaderos y avícolas. Sin embargo, el consumidor norteamericano gasta en alimentos una proporción relativamente baja de su renta en comparación con el mundo en desarrollo. Quienes sufren las consecuencias del encarecimiento en los precios internacionales del maíz y de los productos relacionados son las familias de los países menos desarrollados cuyo acceso a los alimentos básicos se encuentra seriamente en peligro. ${ }^{29}$

\section{El impacto ambiental y los biocombustibles}

El impacto de los biocombustibles en el medio ambiente está relacionado fundamentalmente con los patrones de producción y consumo. Para entender las dimensiones de este impacto los estudios al respecto han adoptado el enfoque de ciclo de vida que comprende la producción agrícola, el transporte y distribución hacia la industria, la transformación industrial, el transporte y distribución hacia el consumo y el consumo propiamente tal. Junto a lo anterior, hay que considerar los impactos ambientales asociados a la producción de determinados insumos y capital productivo como fertilizantes, componentes químicos del proceso industrial, maquinaria agrícola, etc. ${ }^{30}$

La biodiversidad y el cambio climático son los ámbitos que pueden verse más afectados. De acuerdo con ONU-Energía, "los biocombustibles pueden tener efectos positivos o negativos en el uso de la tierra, la calidad de los suelos y del agua y la biodiversidad, dependiendo del tipo de cultivo, de lo que está reemplazando, y de los métodos utilizados para cultivar y cosechar. Los cultivos dedicados a la producción de energía que son aptos para las zonas en las cuales se cultivan -tales como los árboles perennes y las pasturas- pueden minimizar la necesidad de insumos químicos, evitando así parte de la contaminación asociada a la producción de materias primas para la producción de biocombustibles, y dotando del hábitat necesario a aves y otras especies de la fauna silvestre. Las pasturas perennes y la silvicultura de corta rotación también

${ }^{27}$ Paul C. Westcott, "U.S. Ethanol Expansion Driving Changes Throughout the Agricultural Sector", ... op. cit., p. 7.

${ }^{28}$ Backer, A. y Zahniser, S. (2006), "Ethanol Reshapes the Corn Market”, USDA ERS, abril de 2006. Dsiponible en http://www.ers.usda.gov/AmberWaves/April06/Features/Ethanol.htm

29 Incluso el presidente del Banco Mundial, Robert Zoellick, ha dicho que los biocombustibles "contribuyen significativamente" al alza de los precios de los alimentos, contradiciendo así la estimación del Gobierno estadounidense de que los combustibles han contribuido en menos de un 3 por ciento al incremento del precio de los alimentos. Sobre lo sostenido por el Banco Mundial en relación con los precios de los alimentos y la seguridad alimentaria, véase: Banco Mundial, "The Gallagher Review of the indirect effects of biofuels productions" (BM, julio de 2008).

${ }^{30}$ Al respecto, véase: CEPAL (2008), "Consideraciones ambientales en torno a los biocombustibles líquidos”, en División de Desarrollo Sostenible y Asentamientos Humanos, Santiago, Chile, 2008. 
podrían producir un aumento del contenido de carbono de los suelos, en comparación con los cultivos agrícolas anuales". ${ }^{31}$

Por su parte, las emisiones de gases de invernadero en un ciclo de vida completo producidas por la producción de biocombustibles varían ampliamente según los cambios en el uso de la tierra, la elección de la materia prima, las prácticas agrícolas, los procesos de conversión o refinado y las prácticas de uso final. Un buen ejemplo acerca de estos factores lo encontramos en la producción intensiva de maíz en los EE.UU. (cultivo perenne que reemplaza a los cultivos anuales).

Por consiguiente, el impacto ambiental y los riesgos relativos a la disponibilidad y acceso de los alimentos han puesto de relieve la necesidad de reenfocar el problema desde la perspectiva del ciclo de vida así como de los métodos de proceso y producción, los que a su turno deberán encontrar acomodo dentro de las reglas que gobiernan el comercio internacional de los biocombustibles.

En relación con lo anterior, se argumenta que menos barreras comerciales conducirían a una más eficiente asignación de la producción mundial y que dicha reducción enviaría un mensaje acerca del correcto precio a los productores, dirigiendo la futura producción hacia aquellas materias primas más adecuadas en cuanto a eficiencia energética y donde los factores tierra y agua sean más abundante y la competencia con los alimentos sea menos dramática. ${ }^{32}$

\section{IMPLICACIONES DE LOS BIOCOMBUSTIBLES ANTE EL SISTEMA OMC}

\section{A. La "clasificación" en el derecho de la OMC y los biocombustibles}

Según Jackson, ${ }^{33}$ la obligación central del GATT es la concesión arancelaria, que consiste en el compromiso de los miembros ${ }^{34}$ contratantes de no elevar más los aranceles nacionales fijados respecto de un determinado ítem. Este compromiso está incluido en la lista de ese miembro. Los ítem en la Lista son denominados ítem límite (bound) y el

${ }^{31}$ United Nations Commission on Sustainable Development, Sustainable Energy: A Framework for Decision-Makers, op. cit., p. 51 y ss.

${ }^{32}$ Al efecto, véase: Motaal, D.A., “The Biofuels Landscape: Is There a Role for the WTO?”, en Journal of World Trade, $\mathrm{n}^{\circ}$ 42(1), 2008, p. 76.

${ }^{33}$ Jackson, John H., World trade and the law of GATT. A legal analysis of General Agreement on tariffs and Trade, The Bobbs-Merril Company, Charlottesville, Virginia, The Michie Company, Law Publisher, 1969, cap. 10, "Tariff concessions. Their negotiation, and their legal obligation", p. 210.

${ }^{34}$ Cabe señalar que en la nomenclatura de la OMC (a diferencia del GATT de 1947) no se habla de Partes contratantes sino de Miembros contratantes en atención a que no sólo los Estados forman parte de la OMC sino que también territorios aduaneros (por ejemplo, Hong-Kong, Taiwán, etc.) así como Organizaciones Internacionales como es el caso de las Comunidades Europeas. Al efecto, véase Basaldúa, Ricardo X., $L a$ Organización Mundial del Comercio y la Regulación del Comercio Internacional, Lexis Nexis Buenos Aires, 2007, pp. 45 y ss. 
compromiso individual suele denominarse consolidado (binding) o arancel consolidado. Esta obligación se complementa con la llamada cláusula de nación más favorecida $(\mathrm{CN}+\mathrm{F})$ que forma parte del principio de no discriminación (Art. I del GATT) y según el cual cualquier ventaja que un miembro otorgue a otro se hará extensible de manera inmediata e incondicional a todos los demás miembros. ${ }^{35} \mathrm{Nada}$ impide, naturalmente, contemplar aranceles por debajo del consolidado como lo ha hecho Brasil en el caso del etanol, operando por cierto la $\mathrm{CN}+\mathrm{F}$.

La clasificación de los productos ha sido crucial para la operatividad del sistema de límites arancelarios, dado que los países establecen diversos límites, en relación con la $\mathrm{CN}+\mathrm{F}$, para diferentes tipos o clases de productos. Si bien es cierto que los miembros no se encuentran obligados por la OMC en orden a categorizar los productos, la gran mayoría de ellos son miembros también de la Organización Mundial de Aduanas (OMA) en donde sí que se encuentran vinculados en relación con lo que se conoce como Sistema Armonizado (SA). ${ }^{36}$ Consecuentemente, la práctica de la OMC es negociar los aranceles límites en base a dicho sistema. Dentro de este sistema de codificación, a los países les está permitido introducir subclasificaciones; sin embargo, en virtud del art. II del GATT, no pueden modificar el arancel del producto subclasificado más allá del arancel límite, como tampoco pueden violar la obligación del $\mathrm{CN}+\mathrm{F}$ en relación con el tratamiento de los productos similares (like products). ${ }^{37}$

El tratamiento arancelario norteamericano del etanol es un ejemplo ilustrativo de cómo estos principios pueden verse sobrepasados. En efecto, la clasificación del etanol en el SA no distingue entre etanol-combustible de los demás usos del etanol. Sin embargo, los Estados Unidos han impuesto un arancel de 54 céntimos de dólar por galón de etanol para combustible y, por ende, es posible que se encuentre en franca violación con lo dispuesto en el art. II del GATT. ${ }^{38}$

\section{Biocombustibles como bien agrícola y como bien industrial}

Mientras el etanol cae dentro del capítulo 22 del Sistema Armonizado de la OMA y considerado por lo tanto como un bien agrícola en la OMC, el biodiésel cae dentro

${ }^{35}$ Las excepciones a la CN $+\mathrm{F}$ son las contempladas en el art. XX del GATT en relación con los bloques regionales y los tratados de libre comercio. Asimismo, según veremos infra, las preferencias dirigidas a los países en desarrollo o waivers junto al Sistema Generalizado de Preferencias (Cláusula de Habilitación) suponen otro bloque de excepciones a la regla general del art. I del GATT.

${ }^{36}$ El nombre completo, en inglés, es Harmonized Commodity Description and Coding System.

${ }^{37} \mathrm{Al}$ respecto, véase caso Café Español: Spain-Tariff Treatment of Unroasted Coffee, L/5135, adoptado el 11 de junio de 1981, BISD 28S/102. En este caso, lo que ocurrió fue que España subclasificó tipos de cafés para distinguir el café tostado del que no, imponiendo un arancel del 7 por ciento a tres clases de café no tostado mientras que los demás se encontraban libres de impuesto (duty free). Brasil se quejó por violación de la $\mathrm{CN}+\mathrm{F}$ en virtud de que dichos tipos de cafés eran productos similares (like products) a los demás tipos.

${ }^{38}$ Una breve evaluación sobre las posibles disputas comerciales a propósito de la actitud norteamericana como de la UE en relación con los biocombstibles, véase en: Loppacher, Laura J. and William A. Kerr (2005), "Can biofuels become a global industry?: government policies and trade constraints", CEPMLP Internet Journal, Vol. 15, Article 15, www.dundee.ac.uk/cepmlp/journal/html/Vol15/article15_10.html 
del capítulo 38, lo que lo hace un bien industrial. Así pues, las dos clases más relevantes de biocombustibles no compiten en un mismo plano en términos de las reglas de la OMC y por consiguiente son disciplinados bajo regímenes diferentes ${ }^{39}$. En efecto, mientras el etanol encuentra su marco de acción fundamentalmente bajo el Acuerdo sobre Agricultura, el biodiésel se encuentra sujeto al Acuerdo sobre Subvenciones y Medidas Compensatorias. ${ }^{40}$

Todo esto no sólo impide la obtención de información y estadísticas sobre el comercio internacional de biocombustible, sino que impide también los esfuerzos por liberalizar los aranceles de los mismos. Esto conduce a un conjunto de problemas relacionados con la consistencia, la certeza y el principio de no discriminación al momento de aplicar las obligaciones existentes de la OMC. ${ }^{41}$

En consecuencia, no hay una clasificación única para los biocombustibles sino que por el contrario existen diversas clasificaciones que impiden por un lado un mecanismo fiable para llevar adelante estudios estadísticos y, por otro, como veremos, esto influye en los diferentes regímenes que se le aplican a uno y otro biocombustible en materia de aranceles, de subsidios, de flexibilidades, etc. Una complicación adicional a la discusión sobre la clasificación es la posibilidad de entender a los biocombustibles no ya como bienes agrícolas o industriales sino que como bienes (y servicios) ambientales.

\section{Biocombustibles y los bienes y servicios ambientales}

El párrafo 31 (iii) de la Declaración Ministerial de Doha dio inicio a una serie de negociaciones sobre "la reducción o, según proceda, la eliminación de los obstáculos arancelarios y no arancelarios a los bienes y servicios ecológicos" que han sido llevadas a cabo en las Sesiones Especiales del Comité de Comercio y Medio Ambiente (CTE-SS por sus siglas en inglés) y por el Grupo Negociador sobre el Acceso a los Mercados no Agrícolas (NAMA por sus siglas en inglés). Las negociaciones sobre servicios ambientales, por su parte, han sido conducidas dentro de las Sesiones Especiales del Consejo de Comercio y Servicios.

El objetivo detrás de este mandato consiste en que las negociaciones sobre liberalización comercial en bienes y servicios ambientales debían mejorar el mutuo apoyo entre comercio y medio ambiente, sugiriendo un potencial resultado win-win ${ }^{42}$. Es decir, que en

\footnotetext{
${ }^{39}$ Motaal, D.A., “The Biofuels Landscape: Is There a Role for the WTO?”, op. cit, pp. 76-77.

${ }^{40}$ International Institute for Environmental and Development, The multilateral trade and investment context for biofuels: Issues and challenges (IIED, 2007), p. 12. Junto a estos dos acuerdos, también es aplicable a los biocombustibles el de Medidas Sanitarias y Fitosanitarias -MSF- y el de Obstáculos Técnicos al Comercio -OTC-, los que, sin embargo, no serán tratados dada la extensión del presente artículo.

${ }^{41}$ International Food \& Agricultural Trade Policy Council, WTO Disciplines and Biofuels: Opportunities and Constraints in the Creation of a Global Marketplace (IPC Discussion paper, October 2006).

${ }^{42}$ Sobre las negociaciones relativas a bienes y servicios ambientales, véase: UNCTAD (2003), Trade and Environmental Review, Article 2, Vikhlyaev, Alexey, "Environmental goods and services: defining negotiations or negotiating definitions?”; Singh, Sandeep, "Environmental Good Negotiations, Issues and options for ensuring win-win outcomes”, International Institute for Sustainable Development, 2005.
} 
materia de bienes y servicios ambientales, en principio, dada su novedad e indefinición, es susceptible de mejorar las posiciones relativas tanto de los países industrializados como de los países en desarrollo.

Dichas negociaciones se centraron fundamentalmente en definir precisamente qué se entiende por bienes ambientales y los criterios a utilizar. Un tanto complejo fue el criterio de productos medioambientalmente preferibles (EPP por sus siglas en inglés), especialmente aquellos que tendrían que ser definidos en virtud del método de proceso y producción $^{43}$ al que nos hemos referido con anterioridad. Diversos fueron los enfoques dentro de los cuales destaca: i) el sistema de lista basadas en propuestas nacionales; ii) la propuesta de la India denominada "enfoque de proyectos ambientales" (EPA); ${ }^{44}$ y iii) la combinación del EPA con el sistema de lista llamado enfoque integrado, que fuera propuesto por Argentina. ${ }^{45}$

De acuerdo con diversos países, liderados por Brasil, la definición de bienes ambientales cubriría, inter alia, los productos relativos a las energías renovables, dentro de las cuales se incluyen el biodiésel, el etanol y los productos relacionados. ${ }^{46}$ Más allá de los problemas sobre el criterio para determinar qué se considera bien ambiental (las listas suman más de 480 productos), ocurre que el etanol, clasificado como un bien agrícola, quedó fuera del mandato del Grupo Negociador NAMA, responsable de diseñar las modalidades de reducciones arancelarias para los bienes ambientales.

Por su parte, las suspensiones de las negociaciones de Doha, como señala la UNCTAD, probablemente provocarán que los países busquen acuerdos bilaterales o regionales como una herramienta más rápida y predecible para remover barreras arancelarias y no arancelarias afectando el comercio internacional de los biocombustibles así como de las tecnologías relacionadas. ${ }^{47}$

Respecto de los servicios ambientales, cabe nada más señalar que si bien los países en desarrollo han sido tradicionalmente importadores de éstos, en el sector de los biocombustibles alguno de ellos ha desarrollado un expertise que los puede ubicar en posición de exportadores. ${ }^{48}$

Relacionado con el comercio de servicios, en particular los servicios de consultoría, existe un interesante sector que aún no ha sido explotado en relación con los biocombustibles relativo al Mecanismo de Desarrollo Limpio -MDL- contemplado en

${ }^{43}$ United Nations Conference on Trade and Development, The Emerging Biofuels Market: Regulatory..., op. cit., p. 39.

${ }^{44} \mathrm{Al}$ respecto, véase: Un enfoque alternativo para las negociaciones previstas en el apartado III del Párrafo 31. OMC. Doc. TN/TE/W/51 de 3 de junio de 2005.

$45 \mathrm{Al}$ respecto, véanse: Propuesta integradora sobre bienes ambientales para el desarrollo. OMC. Doc. TN/ TE/W/62 de 14 de octubre de 2005.

${ }^{46} \mathrm{Al}$ respecto, véase los siguientes documentos de la OMC: Síntesis de las comunicaciones ambientales. OMC. Doc. TN/TE/W/63 de 17 de noviembre de 2005; y la propuesta de Brasil al respecto: Environmental Goods for Development. OMC. Doc. TN/TE/W/59, de 8 de Julio de 2005, pars. 10-11.

${ }^{47}$ United Nations Conference on Trade and Development, The Emerging Biofuels Market: Regulatory..., op. cit., p. 40.

${ }^{48}$ Ibidem. 
el Protocolo de Kyoto y que eventualmente podría tener alguna significación en los países en desarrollo. ${ }^{49}$

\section{El problema de los productos "similares" (like products) y los biocombustibles}

Puede darse el caso que por razones de política ambiental un miembro de la OMC deseara fomentar el uso de biocombustibles reduciendo para ello los aranceles no obstante mantenerlos cuando el uso de dichos productos no se destine a combustible. Para ello debería, según vimos supra, subclasificar el producto dentro de su nomenclatura doméstica, para lo que no requiere permiso de ninguna naturaleza más allá de la obligación de publicación contenida en el art. X del GATT.

Pues bien, esta opción unilateral, legal hasta acá, podría contestarse bajo la discusión de lo que se entiende por producto similar en relación con la $\mathrm{CN}+\mathrm{F}$. Uno de los problemas a resolver es si la similitud, en este caso, es independiente de las preferencias y percepciones de los consumidores en relación con el uso de ese producto. Si la respuesta fuera negativa, tendríamos que concluir que para este caso los biocombustibles y los productos físicamente similares destinados a usos distintos del combustible deberían considerarse no-similares en tanto que no están compitiendo en el mismo mercado de consumidores. Por lo tanto, un arancel diferente respecto de productos físicamente iguales, en este caso no violaría el art. I del GATT relativo a la discriminación en su acepción externa, es decir, la $\mathrm{CN}+\mathrm{F}^{50}$

Ahora bien, si un miembro de la OMC deseara reducir sus aranceles a las importaciones de biocombustibles basado completamente en razones medioambientales, dicho miembro querrá lógicamente limitar dichas reducciones arancelarias a los biocombustibles que tengan impacto ambiental final positivo de acuerdo con el enfoque del ciclo de vida de ese producto. La pregunta que surge, sin embargo, es si dicha subclasificación sería consistente con la obligación de la $\mathrm{CN}+\mathrm{F}$ en relación con los bienes similares, o si, por el contrario, podría exitosamente desafiarse por otro miembro de la OMC que adujera que sus exportaciones de biocombustibles no gozan del mismo arancel porque no cumple con los criterios de impacto medioambiental del país importador para calificar y acceder a la reducción arancelaria. ${ }^{51}$

Por otro lado, de acuerdo con el art. III del GATT, el principio de no discriminación está orientado hacia dentro, es decir, los miembros no pueden discriminar entre bienes domésticos e importados. Esto significa que al país importador no le está permitido aplicar a los productos extranjeros medidas más onerosas que las impuestas a los productos domésticos similares. Por consiguiente, dentro del contexto de este art. III la

49 Sobre el MDL y los biocombustibles, véase: CEPAL (2007), División de Recursos Naturales e Infraestructura, "Mercado de energías renovables y mercado de carbono en América Latina: estado de la situación y perspectivas”, pp. 43-52; S.J.A. Bakker, CDM and biofuels. Can the CDM assist biofuels production and employment?, informe de Energy Research Center of Netherland, octubre de 2006.

${ }^{50}$ International Food \& Agricultural Trade Policy Council, WTO Disciplines and Biofuels... op. cit.

${ }^{51}$ Ibidem. 
determinación de qué constituye un producto "similar" es un problema crucial desde que la obligación de trato nacional se aplica exclusivamente si dos productos son "similares". 52 Como se puede advertir, el principio de trato nacional es relevante para los biocombustibles en general, pero en particular para las materias primas o feedstocks porque respecto de estos bienes, como el maíz por ejemplo, podría llegarse a distinguir entre producción sustentable de materias primas energéticas de otras materias primas similares no destinadas a la producción de energía.

Por consiguiente, la evaluación acerca de si dos o más productos son similares nos conduce nuevamente al problema sobre si es válido distinguir en función de los PPMs o si por el contrario debería atenderse sólo a las características físicas del bien o bienes. Para Jackson, existe una base textual en el art. III del GATT para la distinción entre producto y proceso de producción aunque dicha distinción debería restringirse con el fin de prevenir abusos proteccionistas. ${ }^{53}$ Por su parte, en la jurisprudencia de la organización relativa al art. XX del GATT (excepciones generales, donde se ubican, inter alia, las de carácter medioambientales así como las de protección a la salud y la vida de las personas, animales y vegetales), parece haber evolucionado hacia interpretar dicho artículo de manera tal que cubre la distinción entre productos basada en la noción de proceso productivo. ${ }^{54}$

El problema se complica aún más si consideramos a los biocombustibles en relación con los combustibles fósiles y el régimen de impuestos que sobre estos últimos pesa en los países. Si ambos tipos de combustibles -los denominados bio y los fósiles- caen dentro de la categoría de bienes similares o sustitutos, en principio la aplicación asimétrica del sistema impositivo (art. III.2 del GATT) podría ser considerada como una violación a las obligaciones del comercio multilateral a menos que se esgriman razones legítimas para sostener dicho sistema discriminatorio. Mientras las razones para tal discriminación han sido la contribución de los biocombustibles para alcanzar los objetivos medioambientales, su impacto ambiental de acuerdo con el ciclo de vida del producto puede no siempre ser positivo, como ocurre con el caso del etanol a base maíz en los Estados Unidos. ${ }^{55}$

${ }^{52}$ United Nations Conference on Trade and Development, The Emerging Biofuels Market: Regulatory..., op. cit., p. 43.

53 Jackson, J.H. (2000). "Comments on Shrimp/Turtle and the Product/Process Distinction”, European Journal of International Law 11, pp. 303-307. Cfr. Cosbey, A. (2001). "The WTO and PPMs: Time to Drop a Taboo", Bridges 5 No. 1-3, at 11-12. Ambos, citados en United Nations Conference on Trade and Development, The Emerging Biofuels Market: Regulatory..., op. cit., p. 43.

${ }^{54}$ Aunque resulte discutible, en el caso Camarones-Estados Unidos, donde lo que se discutía era si la preservación de las tortugas con ocasión de la pesca de camarones con medios que no las pusieran a resguardo de quedar atrapadas en las redes de los pescadores, el Órgano de Apelación del OMC señaló, en el párrafo 121, lo siguiente: "No es necesario suponer que exigir a los países exportadores el acatamiento o la adopción de ciertas políticas (aunque en principio estén cubiertas por alguna de las excepciones) prescritas por el país importador imposibilita a priori la justificación de una medida al amparo del artículo XX. Una interpretación de ese tipo inutiliza la mayoría de las excepciones del artículo XX, si no todas, resultado que repugna a los principios de interpretación que estamos obligados a aplicar". OMC. Doc. WT/DS58/AB/R de 12 de octubre de 1998.

${ }^{55}$ United Nations Conference on Trade and Development, The Emerging Biofuels Market: Regulatory..., op. cit., p. 43. 
4. Preferencias arancelarias bajo el derecho de la OMC (ACP-UE, US-CBI, SGP). Excepciones al principio de no discriminación

Como apunta Basaldúa, "existen diversas excepciones al principio de no discriminación entre las mercaderías importadas que surgen del mismo Acuerdo General”, y que "ellas ponen de manifiesto el pragmatismo que impera en el Acuerdo y que dota de flexibilidad al sistema". ${ }^{56}$ Sin embargo, no todas las excepciones comparten una naturaleza similar. Aquellas que nos ocupan en el presente apartado nacieron como respuesta al hecho de que la situación de los países en desarrollo no había sido contemplada satisfactoriamente en el sistema del GATT de 1947. Según Jouanneau, "el fracaso de la Carta de La Habana constituye un fracaso para el Tercer Mundo, pues el GATT no retiene sino las disposiciones puramente librecambistas de la Carta, dejando de lado aquellas que habrían respondido a las preocupaciones específicas de los países en desarrollo". 57

En el contexto de este esquema de preferencias y excepciones, bajo lo que se conoce como dispensas de obligaciones (waiver clause) - con base legal en el art. XXV del GATT-, nacieron las preferencias extendidas a los países de África, Caribe y el Pacífico (países $\mathrm{ACP}$ ) así como las preferencias existentes bajo la Iniciativa de la Cuenca del Caribe (CBI por sus siglas en inglés). Por su parte, bajo la Cláusula de Habilitación, ${ }^{58}$ surge lo que se conoce como Sistema Generalizado de Preferencias ${ }^{59}$-SGP-, que alude a aquellas preferencias comerciales otorgadas unilateralmente por países desarrollados a productos originarios de países en desarrollo o menos adelantados.

Pues bien, los aranceles con los que ingresa el biocombustible de ciertos países en desarrollo a los países desarrollados se enmarcan dentro de estas excepciones y preferencias comentadas arriba. Así, por ejemplo, el biocombustible de los países ACP entra libre de impuestos a la UE; igual tratamiento reciben los países CBI respecto de los

${ }^{56}$ Basaldúa, Ricardo X., La Organización Mundial del Comercio ... op. cit., pp. 131 y ss. Estas excepciones pueden entenderse también no sólo como un mecanismo que dota de flexibilidad al sistema sino que el modo de convocar a la mayor cantidad de Estados posibles dentro del mismo que, de otro modo, se exponen a quedar fuera de él, lo que no sólo va contra el carácter universal de la Organización sino que relegaría probablemente a dichos Estados a una situación muy desmejorada. En una dirección similar, aunque de naturaleza diversa, juega el sistema de reservas del Derecho internacional público.

57 Ibídem, p. 164 nota la pie $\mathrm{N}^{\circ} 79$.

${ }^{58}$ La Cláusula de Habilitación fue adoptada en la Ronda de Tokio (1973-1979). Por aplicación de sus disposiciones, puede acordarse un trato diferencial y más favorable a los países en desarrollo o, también, entre ellos mismos (Sur-sur), sin que se pueda invocar la aplicación de la $\mathrm{CN}+\mathrm{F}$ ni la reciprocidad. Cabe precisar aquí, que tanto el Cuarto Convenio de Lomé en virtud del cual nace el ACP como el Convenio de Cotonou de 2001 (éste remplazó a aquel) se concluyeron al amparo de la Cláusula de Habilitación y no como waivers.

${ }^{59}$ El SGP no hace, en rigor, honor a su nombre, ya que "no es ni general ni respecto de los países que acuerdan las preferencias, ni respecto de los países en desarrollo beneficiarios, ni incluso respecto de los productos exportados por los países en desarrollo”. Al efecto, véase: Basaldúa, Ricardo X., La Organización Mundial del Comercio... op. cit., p. 169. 
Estados Unidos. ${ }^{60}$ Por su parte, bajo el SGP sólo la UE ha incluido a los biocombustibles provenientes de países en desarrollo; ${ }^{61}$ los Estados Unidos, en cambio, retiraron de este sistema, en los 80, a los biocombustibles.

Lo que interesa destacar, sin embargo, es que bajo el SGP (cuyas reglas especiales se resumen en que tales preferencias deben ser generales, no discriminatorias y la no necesidad de reciprocidad) es posible identificar algunos temas de importancia relativos al desarrollo sostenible o producción sustentable, en el sentido de que es posible condicionar la concesión de la preferencia a estándares de sustentabilidad. El problema puede surgir en cuanto a la posibilidad de distinguir entre distintos países en desarrollo en razón de criterios de sostenibilidad, como se desprende del caso CE-Preferencias Arancelarias. ${ }^{62}$

De acuerdo con Enrique René de Vera, el SGP puede otorgar un marco de discriminación lícita (legal) en contra de la producción insostenible de biocombustibles, ${ }^{63}$ ya que si es posible otorgar preferencias bajo ciertas condiciones -y al efecto cita la negativa de los Estados Unidos a conceder preferencias a países comunistas-, asimismo se pueden aplicar a la producción de biocombustible. De hecho, las exigencias de sostenibilidad ya existen en el marco del esquema del SGP adoptado por la UE aplicado a las maderas tropicales, lo que proveería un modelo a seguir para los biocombustibles. Bajo el SGP de la UE, sólo las maderas que han sido certificadas como sustentables por la Organización Internacional de las Maderas Tropicales reúnen los requisitos para las preferencias arancelarias.

\section{B. Subsidios en el derecho de la OMC y biocombustibles}

La manera en la cual los biocombustibles son clasificados es importante no solo en cuanto a los aranceles concierne sino que también determina la forma en que la OMC disciplina la aplicabilidad de los subsidios domésticos. En efecto, dependiendo del

${ }^{60}$ Cabe señalar que parte del etanol brasileño importado por los Estados Unidos entre de manera directa y otra parte lo hace a través de los países caribeños sorteando de este modo los elevados aranceles norteamericanos. Al respecto, véase: United Nations Conference on Trade and Development, The Emerging Biofuels Market: Regulatory..., op. cit., p. 9.

${ }^{61}$ Sin embargo, recientemente la UE ha excluido del SGP a las exportaciones de biocombustibles de Pakistán, el que suministra el 20 por ciento del etanol importado por la UE. Lo anterior, con motivo de la investigación abierta por la UE de antidumping en contra de este país.

${ }^{62}$ En este caso, el Órgano de Apelación, aunque confirmó la conclusión del Grupo Especial, revocó su razonamiento y constató que no todas las diferencias en el trato arancelario de los beneficiarios del SGP necesariamente constituían un trato discriminatorio. El otorgamiento de distintas preferencias arancelarias a productos originarios de distintos beneficiarios del SGP está autorizado por los términos "no discriminatorio" que figuran en la nota 3 al párrafo 2, siempre que las preferencias arancelarias pertinentes respondan positivamente a una "necesidad de desarrollo, financiera o comercial" concreta y se pongan a disposición, sobre la base de un criterio objetivo, de "todos los beneficiarios que comparten esa necesidad". Pues bien, el Órgano de Apelación sugirió que el significado de "necesidades de desarrollo" podría estar determinado sobre la base de referencias a los instrumentos multilaterales relacionados con el desarrollo sostenible.

${ }^{63}$ René de Vera, E., "The WTO and Biofuels: The possibility of unilateral sustainability requirement", en Chicago Journal of International Law, invierno de 2008. 
biocombustible de que se trate, al Acuerdo sobre Agricultura (AoA por sus siglas en inglés) se le sumarán también las normas del Acuerdo sobre Subvenciones y Medidas Compensatorias (Acuerdo SCM por sus siglas en inglés).

\section{El Acuerdo sobre Subvenciones y Medidas Compensatorias y los biocombustibles}

El concepto de subvención definido en el art. 1.1. del Acuerdo SCM se integra con dos elementos: por una parte, la intervención o la acción pública bajo la modalidad de una contribución financiera, una protección o ganancia o un sostén de precios y, por otra, la ventaja que esta intervención trae aparejada al beneficiario concedido a favor del destinatario. ${ }^{64}$

Por su parte, las subvenciones se clasifican en tres categorías: las prohibidas (Parte II y Anexo I), las recurribles (Parte III) y las autorizadas (Parte IV). ${ }^{65}$ Las subvenciones prohibidas son aquellas subvenciones supeditadas a los resultados de exportación y aquellas supeditadas al empleo de productos nacionales con preferencia a los importados. Las subvenciones recurribles son admitidas en principio, pero si provocan un perjuicio a otro Miembro se hallan sujetas a reclamos.

Teniendo en mente lo anterior, y de acuerdo con el IIED, actualmente existen diversos programas de esta naturaleza y en la medida que la industria se vaya expandiendo se espera que estos programas se sigan desarrollando. Por ejemplo, el Departamento de Agricultura de los Estados Unidos estableció un subsidio para los refinadores que utilicen aceite de soya como materia prima para el biodiésel. Dado que el subsidio sólo está disponible cuando se utiliza aceite de soya como insumo, aquellas firmas que se ven negativamente afectadas por el subsidio, ya sean productores de petróleo o productores de otros insumos que compiten con el aceite de soya, podrían argumentar ante la OMC que el subsidio anula o menoscaba sus beneficios. En caso de que el tema fuera llevado a un panel de la OMC y defendido en forma exitosa, los Estados Unidos tendrían que retirar el subsidio. ${ }^{66}$

De acuerdo con Loppacher, por último, prácticamente todos los subsidios que existen hoy en día en la industria de los biocombustibles satisfacen las condiciones necesarias para ser considerados un subsidio enjuiciable bajo la Parte III del Acuerdo SCM. ${ }^{67}$

\section{El Acuerdo sobre Agricultura y los biocombustibles}

Los Estados suelen contemplar medidas de protección y ayuda al sector agrario con el fin de asegurar una producción suficiente para abastecer al país así como para hacer

${ }^{64}$ Basaldúa, Ricardo X., La Organización Mundial del Comercio... op. cit., p. 311. Cabe recordar que el Acuerdo SCM sólo se aplica a los bienes industriales; por lo tanto, en lo que nos atañe, sólo se aplicaría al biodiésel, no al etanol.

${ }^{65}$ A partir del 1 de enero de 2000, el Acuerdo se refiere sólo a las dos primeras.

${ }^{66}$ International Institute for Environmental and Development, Producción y comercio de biocombustibles $y$ desarrollo sustentable: Los grandes temas (IIED, 2006), p. 33.

${ }^{67}$ Loppacher, Laura J. and William A. Kerr (2005), "Can biofuels become a global industry?..., op. cit. 
frente a las fluctuaciones de los precios internacionales y, también, para preservar la forma de vida de la sociedad rural. ${ }^{68}$ Esto último, es ostensible en el caso de la UE.

La agricultura, además, es para muchos Estados un sector aparte por razones tanto estratégicas como sociológicas. El argumento estratégico se refiere a la independencia en materia alimentaria. El argumento sociológico, por su parte, dice relación con la necesidad de mantener un equilibrio entre las zonas urbanas y las zonas rurales. ${ }^{69}$

Por estas razones, entre otras, fueron acordadas derogaciones a países como la emblemática dispensa a los Estados Unidos por parte del GATT del 47 en el año 1955 (waiver), lo que posibilitó el establecimiento de políticas proteccionistas con barreras no arancelarias como la consagrada en la "Política Agraria Común" -PAC- de la entonces CEE. Esto supuso que la agricultura quedara prácticamente fuera del sistema hasta el GATT de 1994.

Sin embargo, la agricultura volvió al campo de las negociaciones por iniciativa de los propios países desarrollados en 1994 como respuesta a las políticas que sostienen los precios internos o que subvencionan de alguna manera la producción y por lo tanto estimulan el exceso de producción. Esto frena las importaciones o bien da origen a subvenciones a la exportación y a ventas a precios dumping en los mercados mundiales. ${ }^{70}$

Es por esto que en el AoA se distingue entre los programas de ayuda que estimulan directamente la producción y aquellos que se consideran que no tienen ningún efecto directo. Respecto de los primeros, las ayudas caen dentro de la categoría que se denomina compartimento o caja ámbar, por referencia al color ámbar de los semáforos que indica desaceleración. Las medidas que tienen efectos mínimos en el comercio se pueden aplicar libremente y caen dentro de la caja verde. Por último, están permitidos ciertos pagos directos a los agricultores en casos en que se les exija limitar la producción (caja azul).

Pues bien, teniendo presente el breve panorama descrito sobre la agricultura en el comercio internacional, las reglas sobre subvenciones/subsidios del AoA en cuanto a su relevancia respecto de los biocombustibles ${ }^{71}$ han sido resumidas en un documento reciente del Departamento de Agricultura de los Estados Unidos que por su claridad y exactitud es preferible transcribir aquello más relevante:

The classification of measures as "amber box" (in which case they must not have the effect of causing the Member's total support to exceed certain specified ceilings in the AoA) is supposed to be in the first instance based on notification by the subsidizing WTO Member. This kind of notification requirement has proven problematic; a notification of "amber box" measures amounts to an admission that the measures are subject to "amber box" disciplines; thus Members have an incentive not to notify and wait and see whether in fact their measures are challenged in dispute settlement. There

\footnotetext{
${ }^{68}$ Basaldúa, Ricardo X., La Organización Mundial del Comercio... op. cit., p. 231.

69 Ibidem.

${ }^{70}$ Ibidem, p. 238.

${ }^{71}$ Cabe recordar que el AoA se aplica a los biocombustibles toda vez que el etanol es clasificado como un bien agrícola. Por otra parte, el Acuerdo es aplicable en razón de lo que se ha llamado cultivos energéticos; es decir, el cultivo de materias primas agrícolas para la producción de biocombustible.
} 
is thus considerable uncertainty as to whether particular WTO Members are actually operating their agricultural support programs within these aggregate ceilings. In the case of biofuels there is particular uncertainty, because many support programs are structured not as support for individual agricultural products that fall under the AoA but to the biofuels industry; the question then is whether given these latter programs nevertheless operate, at least in part, so as to confer support to agricultural products that are covered by the AoA such that they may fall into the amber box. The stakes are considerable, for if a large number of these measures were considered to be "amber box", the aggregate ceilings to which Members have agreed to might well be broken in certain cases, included those of the US and the EC."72 (Énfasis añadidos).

\section{Conclusiones}

Tras haber descrito el panorama general que se encuentra detrás del sector de los biocombustibles, cabe recordar que a propósito de los problemas que han surgido de la mano con la aparición de la industria y, en particular, con el comercio internacional de este producto, a saber, la seguridad alimentaria y el impacto ambiental, han motivado un llamado a tratar el problema bajo un consenso intergubernamental como la mejor solución al momento de tratar con las políticas involucradas en el tema.

Sin embargo, dicho consenso no parece haberse divisado aún en el horizonte cercano y por consiguiente el Sistema Multilateral de Comercio y en especial el derecho de la OMC aparece como el foro que más puede hablar sobre el punto.

No obstante esta afirmación, dentro de la OMC no se ha tratado el tema de los biocombustibles de forma autónoma y directa y por lo tanto la intención de este artículo ha sido presentar la mayoría de los aspectos que deberían ser tenidos en cuenta a la hora de disciplinar al sector de los biocombustibles y, en ese afán, la literatura ha demostrado que existen múltiples aspectos de difícil solución, más aun cuando el problema exige incorporar la noción de desarrollo sostenible.

En este orden de ideas, lo primero que salta a la vista es la necesidad de una mayor unidad en el tratamiento de los biocombustibles teniendo presente la diversidad intrínseca del producto.

Lo anterior, junto a la noción de sostenibilidad en el comercio internacional son los aspectos más complejos de resolver. Sobre esto último, debe recordarse la necesidad de dar cabida al enfoque comprensivo de ciclo de vida así como a la noción de métodos de proceso y producción, que de una u otra manera entrecruzan las normas del sistema OMC cuando queremos aplicarlas al sector de los biocombustibles.

${ }^{72}$ United States Department of Agriculture, “2007 Farm Hill Theme Paper: Energy and Agriculture”, Washington D.C., agosto de 2006, p. 18. Ejemplos concretos relativos a los subsidios para la producción y consumo de biocombutibles, así como aquellos subsidios relativos a las materias primas para producir biocombustibles y los subsidios para los subproductos creados por la industria del sector, véase en: International Food \& Agricultural Trade Policy Council, WTO Disciplines and Biofuels... op. cit. 
Creemos que si fuera posible introducir de forma sistemática estas nociones, de modo de aplicarlas en su justa medida, es decir, sin que con ellas se encubran restricciones arbitrarias al comercio, ayudaría a resolver los riesgos creados por el sector y ubicaría la producción ahí donde es más eficiente, donde existen las ventajas comparativas más evidentes, sin que por eso se ponga en peligro la seguridad alimentaria y, por otro lado, se colabore con los problemas ambientales como el calentamiento global y la pérdida de biodiversidad.

Cabe esperar que se reinicien las negociaciones de la Ronda de Doha y se dé la oportunidad para tratar el tema de los biocombustibles de manera integrada y abarcadora. Tal vez, los biocombustibles pueden ser la mejor excusa para reiniciar una vez más los procesos de negociación multilateral del comercio y evitar la proliferación de tratados bilaterales que se han transformado en la regla desvirtuando su naturaleza excepcional.

\section{BIBLIOGRAFÍA}

Almada, M., "Análisis de la producción de materias primas para la elaboración de bioetanol y biodiésel, y de estos combustibles, presente y esperada hasta 2020, en países potencialmente proveedores de Chile", Estudio realizado en estadía profesional en la FAO, 2006.

BAnCO MUNDiAL, "The Gallagher Review of the indirect effects of biofuels productions" (BM, julio de 2008).

BACKER, A. Y ZAHNISER, S. (2006)., "Ethanol Reshapes the Corn Market", USDA ERS, abril de 2006.

BasaldúA, Ricardo X., La Organización Mundial del Comercio y la Regulación del Comercio Internacional, Lexis Nexis Buenos Aires, 2007.

CEPAL (2007), División de Recursos Naturales e Infraestructura, "Mercado de energías renovables y mercado de carbono en América Latina: estado de la situación y perspectivas".

CEPAL (2008), "Consideraciones ambientales en torno los biocombustibles líquidos", en División de Desarrollo Sostenible y Asentamientos Humanos, Santiago, Chile, 2008.

Delvaux, B., "Prompting Biofuels in Energy Supply the European Legal Framework", en European Environmental Law Review, 2004.

Energy Policy Research Foundation, Ethanol Part II: Is a Home-Grown Fuel Policy Undermining U.S. Energy Security?, 2007.

FAO, Bioenergía, Seguridad y Sostenibilidad Alimentarias: Hacia el Establecimiento de un Marco Internacional (Roma: FAO, 2008. Doc. HLC/08/INF/3).

INSTITUTO INTERAMERICANO DE COOPERACIÓN PARA LA AGRICULTURA, Estrategias para construir una plataforma de cooperación horizontal sobre agroenergía y biocombustibles (IICA, 2006).

International Energy Agency, World Energy Outlook 2006 (OECD/AIE 2006).

International Food \& Agricultural Trade Policy Council, WTO Disciplines and Biofuels: Opportunities and Constraints in the Creation of a Global Marketplace (IPC Discussion paper, October 2006).

International Institute for Environmental and Development, Producción y comercio de biocombustibles y desarrollo sustentable: Los grandes temas (IIED, 2006).

INTERNATIONAL INSTITUTE FOR ENVIRONMENTAL AND DEVELOPMENT, The multilateral trade and investment context for biofuels: Issues and challenges (IIED, 2007).

Jackson, John H., World trade and the law of GATT. A legal analysis of General Agreement on tariffs and Trade, The Bobbs-Merril Company, Charlottesville, Virginia, The Michie 
Company, Law Publisher, 1969, cap 10, "Tariff concessions. Their negotiation, and their legal obligation".

Jank Marcos, et. al., EU and US Policies on Biofuels: Potential Impacts on Developing Countries (Washington, D.C.: German Marshall Fund of the United States, 2007).

Loppacher, Laura J. and William A. Kerr (2005), "Can biofuels become a global industry?: government policies and trade constraints”, CEPMLP Internet Journal, Vol. 15, Article 15.

MotAal, D.A., "The Biofuels Landscape: Is There a Role for the WTO?", en Journal of World Trade, $\mathrm{N}^{\circ}$ 42(1), 2008.

Paul C. Westcott, "U.S. Ethanol Expansion Driving Changes Throughout the Agricultural Sector," Amber Waves, U.S. Department of Agriculture, septiembre de 2007.

René DE Vera, E., "The WTO and Biofuels: The possibility of unilateral sustainability requirement", en Chicago Journal of International Law, invierno de 2008.

SINGH, SANDEEP, "Environmental Good Negotiations, Issues and options for ensuring win-win outcomes", International Institute for Sustainable Development, 2005.

S.J.A. BAKKER, CDM and biofuels. Can the CDM assist biofuels production and employment?, informe de Energy Research Center of Netherland, octubre de 2006.

UnCTAD (2003), Trade and Environmental Review, Articule 2, Vikhlyaev, Alexey., "Environmental goods and services: defining negotiations or negotiating definitions?".

United Nations Conference on Trade and Development, The Emerging Biofuels Market: Regulatory, Trade and Development Implications (Geneva: UNCTAD, 2006).

United Nations Commission on Sustainable Development, Sustainable Energy: A Framework for Decision- Makers (Mayo 2007).

United States Department of Agriculture, "2007 Farm Hill Theme Paper: Energy and Agriculture”, Washington D.C., agosto de 2006. 